\title{
QoS Support in ARINC 664 P8 Data Networks: ATN Applications Over TCP/IP Ground-to-Ground Subnetworks*
}

\author{
Haowei Bai ${ }^{\dagger}$ \\ Honeywell Defense and Space Electronics Systems \\ Glendale, AZ 85308, USA \\ Mohammed Atiquzzaman \\ University of Oklahoma, Norman, OK 73019-6151, USA \\ and \\ William Ivancic $\$$ \\ NASA John H. Glenn Research Center at Lewis Field, Cleveland, OH 44135, USA
}

\begin{abstract}
Aeronautical Telecommunication Network (ATN) has been developed by International Civil Aviation Organization (ICAO) to integrate air-ground and ground-ground data communications for aeronautical applications into a single network serving air traffic control and aeronautical operational communications. The current ICAO ATN model specifies a standalone network with its own protocols, which requires building an expensive separate network for air-ground and ground-ground communications. However, the cost of building ATN can be reduced if it can use existing networks wherever possible, such as the Internet. ARINC 664 Part 8 committee has been developing an approach for inter-operation of ATN applications over the commercial TCP/IP networks. To carry time critical information required for aeronautical applications, ATN provides different Quality of Services (QoS) to applications. Although the current Internet does not provide QoS, Internet Engineering Task Force (IETF) developed the Differentiated Services (DiffServ) network to provide differential QoS to users of next generation data networks. The objective of this paper is to investigate the possibility and conduct a proof-of-concept evaluation of providing QoS to ATN applications over the DiffServ network in the next generation Internet. Our results show that the QoS requirements of ATN applications for ground-to-ground communications can be successfully satisfied when ATN is run over a DiffServ backbone.
\end{abstract}

\section{Introduction}

TNERNATIONAL Civil Aviation Organization (ICAO) has developed ATN as a commercial networking infrastructure to integrate air-ground and ground-ground data communication into a single network for air traffic control

Received 8 July 2005; revision received 8 July 2005; accepted for publication 22 February 2006. Copyright (@) 2006 by the American Institute of Aeronautics and Astronautics, Inc. All rights reserved. Copies of this paper may be made for personal or internal use, on condition that the copier pay the $\$ 10.00$ per-copy fee to the Copyright Clearance Center, Inc., 222 Rosewood Drive, Danvers, MA 01923; include the code 1542-9423/04 \$10.00 in correspondence with the CCC.

* The work reported in this paper was partially supported by NASA Grant NAG3-2318.

$\dagger$ Honeywell Defense and Space Electronics Systems, 19019 N 59th Avenue, Glendale, AZ 85308, USA. haowei.bai@honeywell.com

$¥$ School of Computer Science, University of Oklahoma, Norman, OK 73019-6151, USA. atiq@ ou.edu

$\S$ NASA Glenn Research Center, 21000 Brookpark Rd. MS 54-8, Cleveland, OH 44135, USA. william.d.ivancic@grc.nasa.gov 
and aeronautical operational communications. ${ }^{1}$ One of the ATN objectives is to accommodate different QoS required by air traffic management, airline operation control, airline administrative communications, airline passenger communications, and entertainment applications. In ATN, priority has the essential role of ensuring that high priority safety related and time critical data are not delayed by low priority non-safety data, especially when the network is overloaded with low priority data. The network layer of ATN, called the connectionless network protocol (CLNP), routes packets from source to destination. Routers read the information in CLNP headers, and offer preferential treatment to packets in terms of prioritizing the packet relative to other packets. CLNP is a connectionless unreliable protocol which does not provide guarantee of data delivery. An end-to-end transport layer protocol, called transport protocol class 4 (TP4), is required to guarantee reliable delivery of data between end points.

Current commercial Internet only offers end-to-end best-effort service and therefore is not suitable for carrying data which has priority requirements. However, the rapid commercialization of the Internet has given rise to demands for QoS over the Internet. QoS is generally implemented by different classes of service contracts for different users. A service class may provide low-delay and low-jitter services for customers who are willing to pay a premium price to run high-quality applications, such as, real-time multimedia. Another service class may provide predictable services for customers who are willing to pay for reliability. The best-effort service provided by current Internet will remain for those customers who need only connectivity.

IETF has proposed a few models to meet the demand for QoS for next generation Internet. Notable among them are Integrated Services (IntServ) ${ }^{2}$ and Differentiated Services (DiffServ). ${ }^{3}$ IntServ is characterized by resource reservation. Before data is transmitted, applications must set up paths and reserve resources along the path. This gives rise to the scalability issue in core routers of large networks. DiffServ overcomes the above scalability issue, and accommodates the various service guarantees required for time critical applications. DiffServ utilizes six bits in the type of service (TOS) field of the IP header to assign each packet a differentiated service code point (DSCP) for being eligible for a particular per-hop behavior (PHB). Packets are grouped into behavior aggregates (BA) and routed based on their DSCP codes. Diffserv does not require significant changes to the existing infrastructure or too many additional protocols.

The time critical information carried by ATN and the QoS required by ATN applications have led to the development of ATN as an expensive standalone network. Significant cost saving can be achieved if ATN could be inter-operated with the next generation Internet, as shown in Fig. 1. ARINC 664 Part $8^{\dagger}$ committee has defined three transition strategies to address the inter-operation of ATN protocols and services with TCP/IP based Internet infrastructure for ground-to-ground communications. ${ }^{4}$ Due to criticality and security requirements for air-toground communications, currently ICAO has no plans to transition air-to-ground communications to TCP/IP based architecture.

- CLNP over IP based ground infrastructure using SNDCF: In this architecture, ATN applications use CLNP in the aircraft and communicate to the ground ATN router using ATN-ground subnetwork(s). On the ground, ATN ground router interfaces to IPv4/IPv6 sub-network(s). CLNP packets are encapsulated in IP packets using the sub-network dependent convergence function (SNDCF). There are no certification issues as all changes are below the transport layer. But this architecture needs significant changes on the ground-ground infrastructure.

- CLNP-IP router for ground infrastructure: In this architecture, the aircraft and air-ground network(s) are fully ATN compliant. The ground-ground communication is based on pure IPv6 with a CLNP-IPv6 router to map CLNP functions to IP functions. This architecture does not have certification issues as all changes are to be made below the transport layer. The concept of CLNP-IP router provides modularity and reduces complexity for network deployment.

\footnotetext{
${ }^{\dagger}$ Aeronautical Radio, Inc. (ARINC) is a world leading provider of transportation communications and systems engineering solutions for aviation, airports, defense, government, and transportation. The Airlines Electronic Engineering Committee (AEEC) is an international standards organization, comprising major airline operators and other airspace users. The AEEC establishes consensus-based, voluntary form, fit, function, and interface standards that are published by ARINC and are known as ARINC Standards. ARINC 664 is a standard for Aircraft Data Networks, of which, a working project ARINC 664 Part 8, Interoperation with Non-IP Protocols and Services, provides guidelines for development of aeronautical applications and services other than those defined in TCP/IP family of protocols so they may be transported over the TCP/IP network.
} 


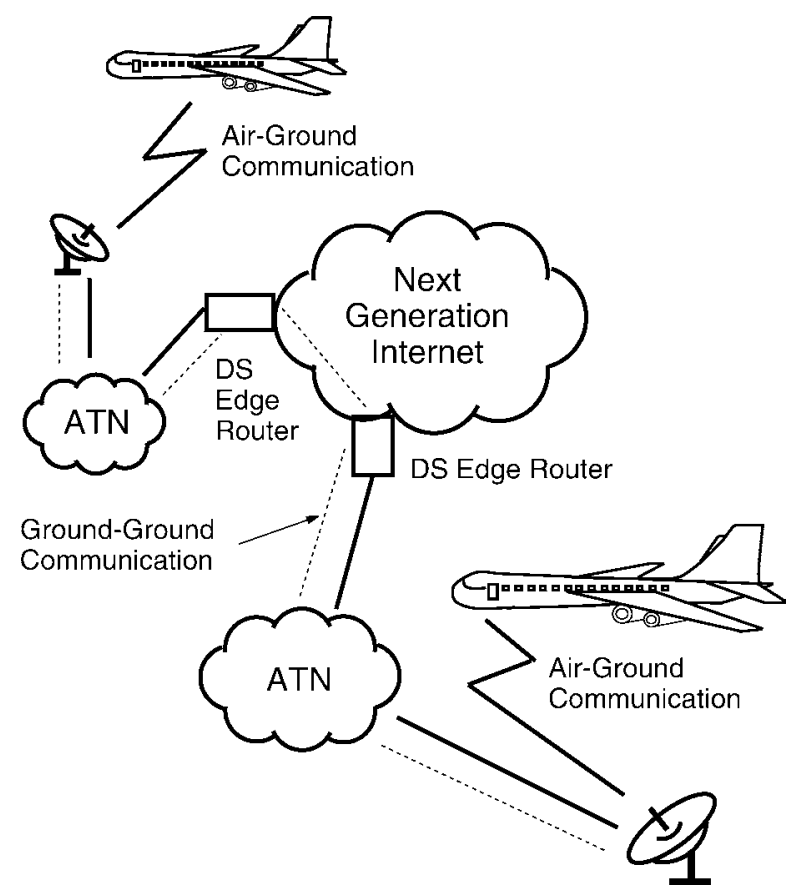

Fig. 1 Interconnection between ATN and Differentiated Services.

- Dual stack operation: In this approach, all communication nodes in the network have to use dual protocol stack. The ATN applications are made to operate over IPv6 by adding a modified dialog services (DS) that uses IPv6 communication mechanism. This architecture requires significant changes to the network and may require high-level certification for IP network components.

The approach of adding CLNP-IP router for ground infrastructure provides unique cost effectiveness. The only modification required is addition of an appropriate mapping function at the edge routers (CLNP-IP routers) between two network domains. This architecture will lead to aerial ATN islands being connected by terrestrial IP networks. However, ARINC 664 Part 8 has not provided a detailed architecture for CLNP-IP router.

There have been many studies to assess the capabilities of IP protocol suite against the requirements of ATN applications. The IPSky project ${ }^{5}$ experimentally evaluated the suitability of IPv6 for use in ATN networks. Their evaluation, based on the QoS, mobility, reliable multicast, and address translation features of IPv6, demonstrated that IPv6 is suitable for aeronautical communications.

Dhas et al. ${ }^{6}$ have investigated the possibility of the use of IP in avionics. Their report reinforces the fact that TCP/IPv6 can support ATN applications. Their cost analysis drew the conclusion that transporting ATN application data over TCP/IPv6 would significantly reduce the costs (up to 28 times).

Similar conclusions to those reported in ${ }^{5,6}$ were also reported by Netto et al. ${ }^{7}$ and Crouzard et al. ${ }^{8}$ They noted that the aviation community can achieve operational benefits if TCP/IP protocol suite is used to implement ATN. They have also presented a number of architectures for implementing ATN over the TCP/IP protocol stack, addressing the issues of mobility, security and QoS, and outlined a strategy for gradually replacing CLNP with IP on the ground.

The work described above ${ }^{5-8}$ which recommend the use of TCP/IP protocol suite for implementing ATN are based on architectural and/or cost analysis. Quantitative performance comparison was not the focus of any of the above studies. However such a quantitative performance evaluation study is required before a decision can be made to implement any of the techniques.

The objective of this paper is to propose a proof-of-concept architecture for CLNP-IP router to ensure QoS delivery from ATN domain to IP domain, and perform quantitative performance evaluation using computer simulations. Based on the similarity between an IP packet and an ATN packet, our approach is to add a mapping function to the existing 
DiffServ edge router so that the traffic flows coming from ATN domain can be appropriately mapped into the corresponding BA of DiffServ, and then marked with the appropriate DSCP for routing in DiffServ domain. With the mapping function, the DiffServ router performs the functionality of CLNP-IP router specified by ARINC 664 Part 8. Our simulation results show that, without making any significant changes to the ATN or DiffServ infrastructure, and without any additional protocols or signaling, the mapping function is able to appropriately deliver QoS requirements for ATN applications over a DiffServ network.

The rest of this paper is organized as follows. In Sections II and III, we briefly review ATN and DiffServ, respectively. We present our approach to interconnecting ATN with DiffServ in Section IV, and the simulation configuration for evaluations in Section V. In Section VI, we analyze our simulation results to show that QoS can be provided to applications in the ATN domain. Concluding remarks are finally given in Section VII.

\section{Aeronautical Telecommunication Network (ATN)}

In the early 1980s, ICAO recognized the increasing limitations of the present air navigation systems and the need for improvements to take civil aviation into the $21^{\text {st }}$ century. The need for changes in the current global air navigation system is due to two factors:

- The present and growing air traffic demand which the current system will be unable to handle;

- Global consistency in the provisioning of air traffic services during the progression towards a seamless air traffic management system.

The above factors gave rise to the concept of the Aeronautical Telecommunication Network. ${ }^{9}$

ATN is both a ground-based network providing communications between ground-based users, and an air-ground network providing communications between airborne and ground users. It was always intended that ATN should be built on existing technologies instead of inventing new ones. The Internet approach was seen as the most suitable approach, and was therefore selected as the basis for the ATN. ATN consists of end systems, intermediate systems, ground-ground subnetworks, and air-ground subnetworks as shown in Fig. 1.

\section{A. Priority in ATN}

ATN has been designed to provide high reliability/availability by ensuring that there is no single point of failure, and permitting the availability of multiple alternative routes to the same destination with dynamic switching between alternatives. To ensure that low priority data does not impede the flow of high priority data, every ATN user data is given a relative priority on the network. The purpose of priority is to signal the relative importance and (or) precedence of data, such that when a decision has to be made as to which data to act first, or when contention for access to shared resources has to be resolved, the decision or outcome can be determined unambiguously and in line with user requirements both within and between applications.

Priority in ATN is signaled separately by the application in the transport layer, network layer, and in ATN subnetworks, which gives rise to Transport Priority, Network Priority and Subnet Priority. ${ }^{10}$ Network priority is used to manage the access to network resources. During periods of high network utilization, higher priority Network Protocol Data Units (NPDU) may therefore be expected to be more likely to reach their destination (i.e. less likely to be discarded by a congested router), and to have a lower transit delay (i.e. more likely to be selected for transmission from an outgoing queue) than lower priority packets. In this paper, as required by the CLNP-IP router approach, we focus on network priority which determines the sharing of limited network resources.

\section{B. ATN Packet Format}

Figure 2 shows the format of an ATN packet, and the similarity between ATN packet and IP packets. It is seen that the fields of the two packets carry similar information, and can be easily mapped to each other. This provides the possibility for mapping ATN to DiffServ to achieve the required QoS when they are interconnected.

The NPDU header of an ATN packet contains an option part, including an 8-bit field named Priority which indicates the relative priority of NPDU. ${ }^{1}$ Values 00000001 through 00001110 are to be used to indicate the priority in an increasing order. A value of 00000000 indicates normal priority. 


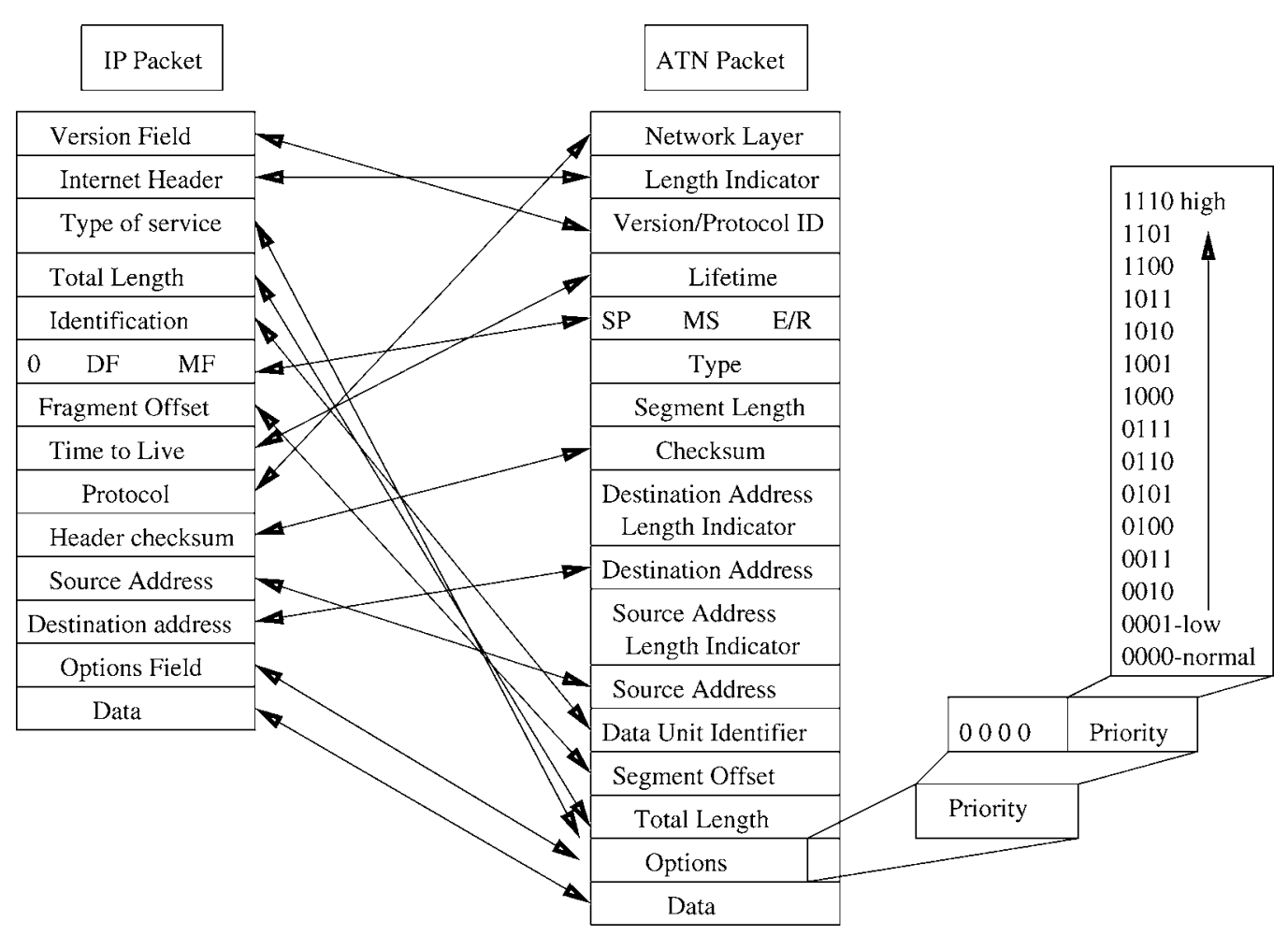

Fig. 2 Similarity between an IP packet and an ATN packet.

\section{Differentiated Services}

DiffServ is intended to enable the deployment of scalable service discrimination in the Internet without the need for per-flow state and signaling at every hop. The premise of DiffServ networks is that routers in the core network handle packets from different traffic streams by forwarding them using different PHBs. The PHB to be applied is indicated by a DSCP in the IP header of the packet. ${ }^{11}$ The advantage of such a mechanism is that several different traffic streams can be aggregated to one of a small number of BA which are each forwarded using the same PHB at the router, thereby simplifying the processing and associated storage. ${ }^{12}$ There is no signaling or processing since QoS is invoked on a packet-by-packet basis. ${ }^{12}$

The DiffServ architecture is composed of a number of functional elements, including a small set of per-hop forwarding behavior, packet classification function, and traffic conditioning function which includes metering, marking, shaping and policing. The functional block diagram of a typical DiffServ router is shown in Fig. 3. ${ }^{12}$ This architecture provides Expedited Forwarding (EF) service, Assured Forwarding (AF) service, as well as best-effort (BE) service as described below.

\section{A. Expedited Forwarding}

Expedited forwarding (EF), also described as Premium Service, provides a low loss, low latency, low jitter, assured bandwidth, end-to-end service for customers. ${ }^{13}$ Loss, latency and jitter are caused by queuing experienced by traffic while transiting the network. At each transit node, the aggregate of the EF traffic's maximum arrival rate must be less than its configured minimum departure rate to ensure there is almost no queuing delay for premium packets. Packets exceeding the peak rate are shaped by traffic conditioners to bring the traffic into conformance.

\section{B. Assured Forwarding}

Assured forwarding (AF) provides a reliable services for customers, even at presence of network congestion. Classification and policing are first done at the edge routers of the DiffServ network. The AF traffic is considered 


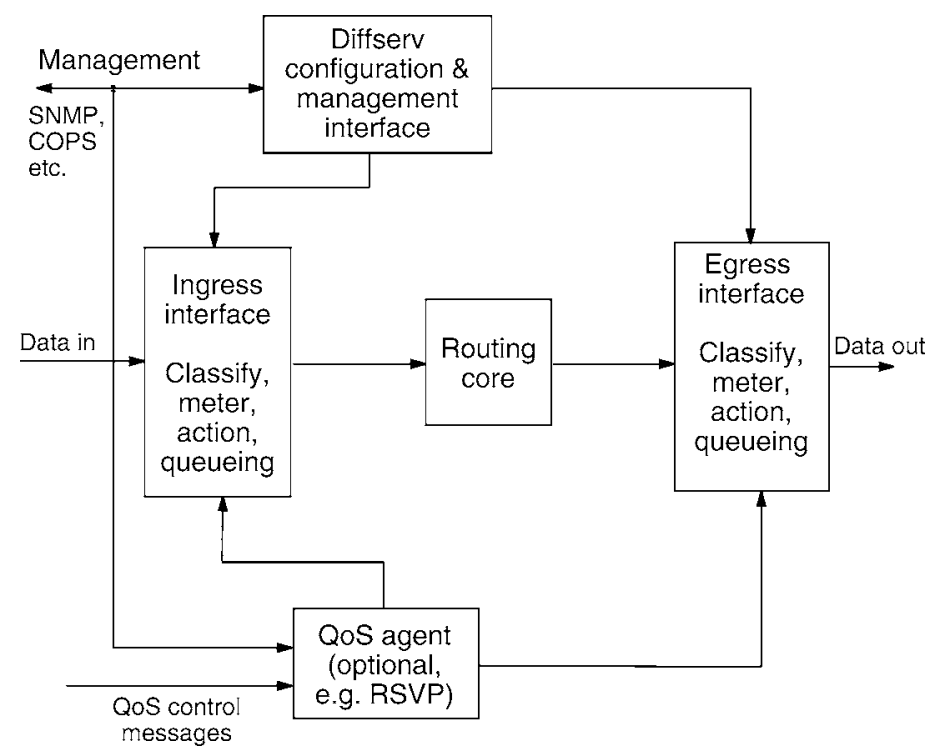

Fig. 3 Functional block diagram of a DiffServ router.

in-profile if the traffic does not exceed the bit rate allocated for the service; otherwise, the excess packets are considered out-of-profile. The in-profile packets should be forwarded with high probability, and out-of-profile packets are delivered with lower probability. Since the network does not reorder packets that belong to the same microflow, all packets, irrespective of whether they are in-profile or out-of-profile, are put into an assured queue to avoid out-of-order delivery.

Assured forwarding delivers packets in four independently forwarded AF classes. Each class is allocated a configurable minimum amount of buffer space and bandwidth. Each class is in turn divided into different levels of drop precedence. In presence of network congestion, the drop precedence determines the relative importance of packets within the AF class. Figure $4^{14}$ shows four different AF classes with three levels of drop precedence.

\section{Best Effort}

This is the default service available in DiffServ, and is also deployed by the current Internet. It does not guarantee any bandwidth to customers, but can only get the bandwidth available. Packets are queued when buffers are available and dropped when resources are over committed.

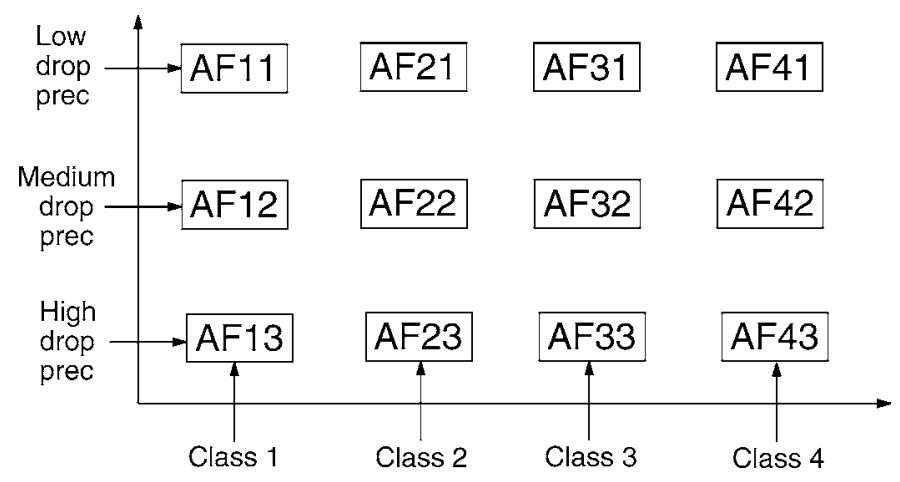

Fig. 4 AF classes with drop precedence levels. 


\section{ATN Over Differentiated Services}

We mentioned in Section I that, as one of strategies to interconnecting ATN and TCP/IP recommended by ARINC 664 Part 8, CLNP-IP router for ground infrastructure offers unique cost effectiveness and no certification hassles. In this approach, the aircraft network architecture is based on the ATN TP4/CLNP, and communicates with the ground router using any one of the ATN sub-networks. The ground-ground infrastructure is based on IPv6 with the ground end-systems running ATN applications over the IPv6 protocol stack. This approach requires a CLNP/IPv6 router function that has to map the CLNP functions to corresponding IPv6 functions in the air-ground direction, and map the IPv6 functions to CNLP functions in the ground-air direction. The result is a pure IPv6 ground network, which moves ATN protocols (CLNP) to the ATN air-ground routers. There is no needs to change the aircraft network.

The main question that we would like to answer in this paper is: can the PHB treatment of packets along the path in the DiffServ domain approximate the QoS offered in the ATN based aircraft network and air-ground network? To answer this question, we added a mapping function at the CLNP-IP router between ATN and DiffServ domain. The architecture of a CLNP-IP router with mapping function is shown in Fig. 5. Packets with different priorities from the ATN domain are first mapped to the corresponding PHBs in the DiffServ domain by appropriately assigning a DSCP according to the mapping function. Packets are grouped into BAs, and routed in the DiffServ domain where they receive treatments based on their DSCP codes. Table 1 shows an example mapping function which has been used in our simulation. Packets with normal priority in ATN domain are mapped to BE PHBs in DiffServ domain. Similarly, medium priority ATN packets are mapped to AF11 PHBs; high priority ATN packets are mapped to EF PHBs.

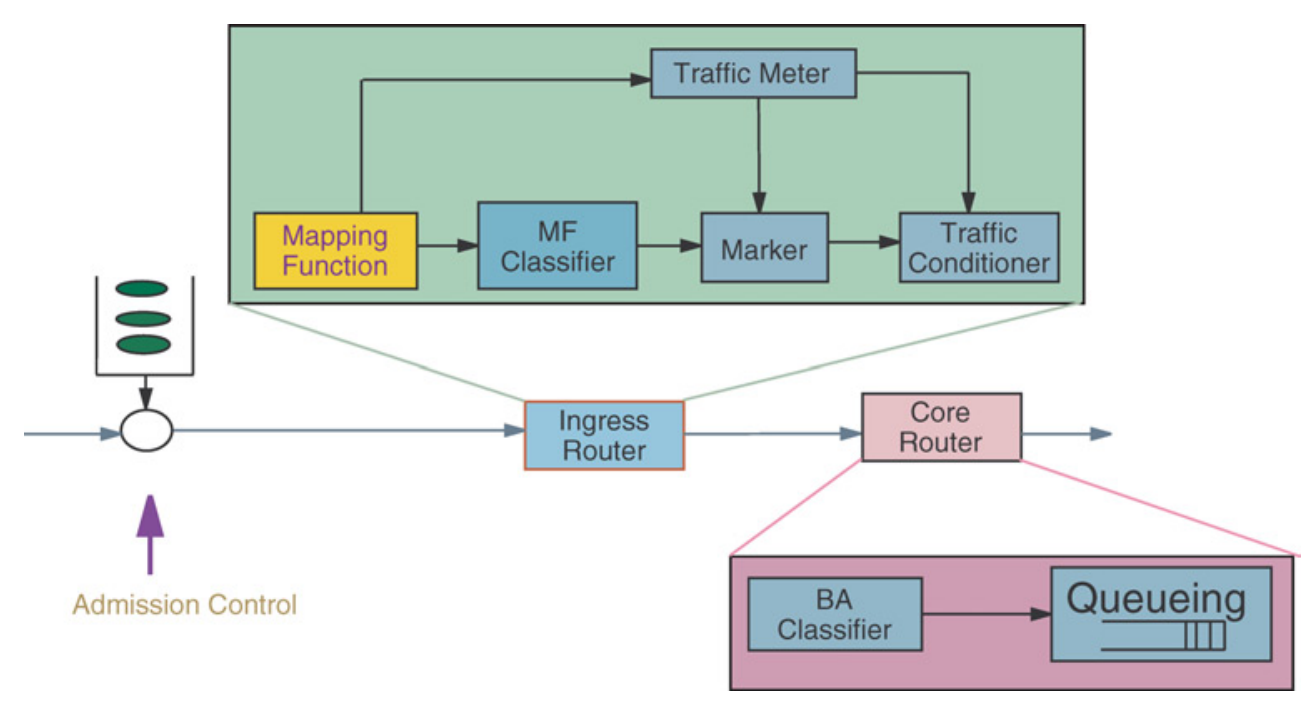

Fig. 5 A CLNP-IP router with mapping function for ATN over Differentiated Service.

Table 1 An example mapping function used in our simulation.

\begin{tabular}{lllc}
\hline ATN Priority Code & Priority & PHB & DSCP \\
\hline 00000000 & Normal & BE & 000000 \\
00000111 & Medium & AF11 & 001010 \\
00001110 & High & EF & 101110 \\
\hline
\end{tabular}




\section{Performance Evaluation Methodology}

To test the effectiveness of our proposed mapping function in CLNP-IP router and to determine how much QoS can be provided to ATN applications, we carried out simulations using the $n s 2$ simulation tool from Berkeley.

\section{A. Network Topology}

The network topology used in our simulation is shown in Fig. 6. Ten ATN sources were used in our simulation. Two sources generating air traffic management data have high priority. Three airline administrative sources have medium priority. Five entertainment sources are set to have normal priority. Ten ATN sinks served as destinations for the ATN sources.

All the links in Fig. 6 are labeled with a (bandwidth; propagation delay) pair. For simplicity of implementation and simulation, the mapping function shown in Table 1 was integrated into the CLNP-IP router. This simulation configuration represents a proof-of-concept ATN over TCP/IP architecture with CLNP-IP router for ground infrastructure, recommended by ARINC 664 Part 8 specification.

Inside the CLNP-IP router, EF queue was configured as a simple Priority Queue with Tail Drop. AF queue was configured as RIO queue and BE queue as a $\mathrm{RED}^{15}$ queue. The queue weights of $\mathrm{EF}, \mathrm{AF}$ and BE queues were set to $0.4,0.4$ and 0.2 respectively. Since the bandwidth of the bottleneck link between two CLNP-IP routers is $5 \mathrm{Mb}$, the above scheduling weights imply bandwidth allocations of $2 \mathrm{Mb}, 2 \mathrm{Mb}$ and $1 \mathrm{Mb}$ for the EF, AF and BE links respectively during periods of congestion at the edge router.

\section{B. Design of Experiments}

We use the following four simulation cases to determine QoS obtained by ATN sources.

- Case 1: No congestion: Traffic generated by each of the high, medium and normal priority sources were set to $1 \mathrm{Mb}, 0.666 \mathrm{Mb}$ and $0.2 \mathrm{Mb}$ respectively. According to the network configuration described in Section $\mathrm{V}$, two high priority sources, three medium priority sources and five normal priority sources are generating $2 \mathrm{Mb}, 2 \mathrm{Mb}$ and $1 \mathrm{Mb}$ traffic respectively. The amount of traffic for each source is equal to the corresponding

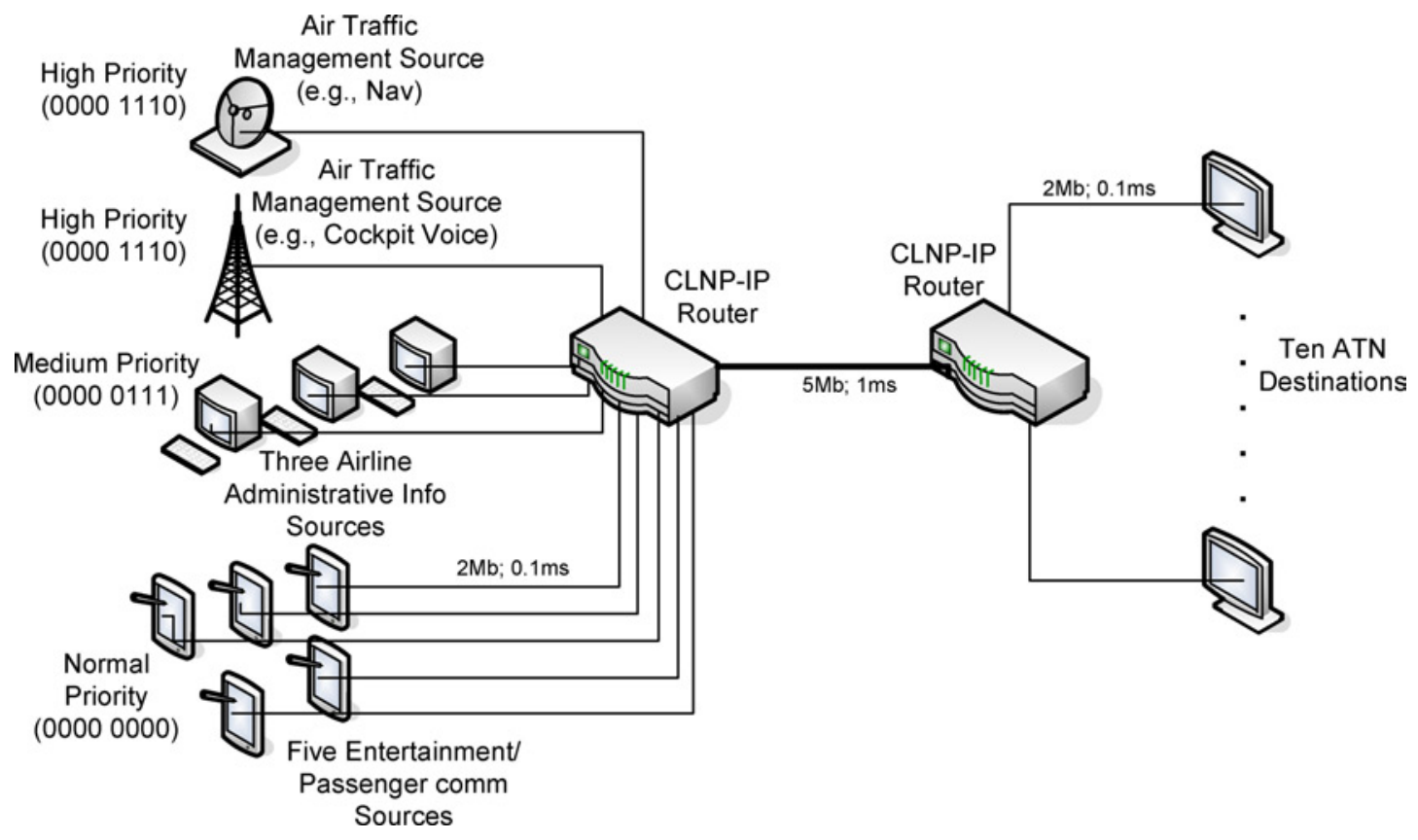

Fig. 6 Network topology for simulation evaluation. 
scheduled link bandwidth assigned by scheduler described in Section V. Under this scenario, there should not be any significant congestion at the CLNP-IP router.

- Case 2: Normal priority traffic gets into congestion: The source rate of each high, medium and normal priority sources was set to $1 \mathrm{Mb}, 0.666 \mathrm{Mb}$ and $0.6 \mathrm{Mb}$ respectively. According to the network configuration described in Section $\mathrm{V}$, the total amount of traffic generated by five normal priority sources is $3 \mathrm{Mb}$. The amount of high and medium priority traffic are still equal to the corresponding output link bandwidth assigned by scheduler described in Section V. However, the amount of normal priority traffic is greater than its corresponding scheduled link bandwidth. Under this scenario, the normal priority traffic gets into congestion at the CLNP-IP router.

- Case 3: Medium priority traffic gets into congestion: Traffic generated by each of the high, medium and normal priority sources were set to $1 \mathrm{Mb}, 1.333 \mathrm{Mb}$ and $0.2 \mathrm{Mb}$ respectively. According to the network configuration described in Section $\mathrm{V}$, three medium priority sources are generating $4 \mathrm{Mb}$ traffic now. Similar to Case 2, the amount of high and normal priority traffic are still equal to the corresponding output link bandwidth assigned by scheduler described in Section V. However, the amount of traffic of medium priority is greater than its corresponding scheduled link bandwidth. Under this scenario, the medium priority traffic gets into congestion at the CLNP-IP router.

- Case 4: Both medium and normal priority traffics get into congestion: Traffic generated by each of the high, medium and normal priority sources were set to $1 \mathrm{Mb}, 1.333 \mathrm{Mb}$ and $0.6 \mathrm{Mb}$ respectively. In this case, two high priority sources, three medium priority sources and five normal priority sources are generating traffic of $2 \mathrm{Mb}, 4 \mathrm{Mb}$ and $3 \mathrm{Mb}$ respectively. The amount of high priority traffic is still equal to the corresponding scheduled link bandwidth assigned by scheduler described in Section V. However, both medium priority traffic and normal priority traffic are greater than their corresponding scheduled link bandwidth. Under this scenario, both medium and normal priority traffics get into congestion at the CLNP-IP router.

\section{Simulation Results}

Table 2 shows the goodput of each ATN source for four different cases described in Section B. Table 3 shows the drop ratio measured at the scheduler for four cases of the three different types of ATN sources. The queuing delay and jitter can be evaluated from Figs 7, 8, 9 and 10 show the queue size for each of the four cases.

Case 1 is an ideal case. Each type of source (high, medium and normal priority sources) generates traffic at the rate equal to the bandwidth assigned by the scheduler. Therefore, there is no significant network congestion at the CLNP-IP router. As seen in Table 2, the goodput of each source is almost the same as its traffic generating rate. From Table 3, the drop ratio of each type of sources is zero. Figure 7 shows the queuing performance of each queue. Because this is an ideal case, the size of each queue is very small. The high priority (mapping to EF PHBs, according to our proposed mapping function) queue has the smallest average queue size; The normal priority (mapping to BE) queue has the largest jitter.

Table 2 Goodput of each ATN source (Unit: Kb/S).

\begin{tabular}{lccccc}
\hline Sources & & Case 1 & Case 2 & Case 3 & Case 4 \\
\hline High priority Sources & Source 0 & 999.9990 & 999.9990 & 999.9990 & 999.9990 \\
& Source 1 & 999.9990 & 999.9990 & 999.9990 & 999.9990 \\
Medium priority Sources & Source 2 & 666.6660 & 666.6660 & 668.2409 & 668.4719 \\
& Source 3 & 666.6660 & 666.6660 & 667.3379 & 667.5270 \\
& Source 4 & 666.6660 & 666.6660 & 664.4189 & 663.9990 \\
Normal priority Sources & Source 5 & 200.0039 & 199.6469 & 200.0039 & 199.4790 \\
& Source 6 & 200.0039 & 201.8520 & 200.0039 & 201.9780 \\
& Source 7 & 200.0039 & 202.4190 & 200.0039 & 201.6840 \\
& Source 8 & 199.9830 & 199.8779 & 199.9830 & 200.4660 \\
& Source 9 & 200.0039 & 196.2030 & 200.0039 & 196.3920 \\
\hline
\end{tabular}


BAI, ATIQUZZAMAN, AND IVANCIC

Table 3 Drop ratio of ATN traffic.

\begin{tabular}{lcccc}
\hline Type of traffic & Case 1 & Case 2 & Case 3 & Case 4 \\
\hline High priority Traffic & 0.000000 & 0.000000 & 0.000000 & 0.000000 \\
Medium priority Traffic & 0.000000 & 0.000000 & 0.499817 & 0.499834 \\
Normal priority Traffic & 0.000000 & 0.665638 & 0.000000 & 0.665616 \\
\hline
\end{tabular}

In Case 2, we increased the traffic generating rate of normal priority sources, keeping the rates of the other two types of traffic unchanged. The traffic generating rate of each normal priority source is set to $0.6 \mathrm{Mb}$. In this case, the normal priority traffic gets congested. As shown by Table 3, the drop ratio of normal priority traffic is greatly increased. However, drop ratio for the other two sources still remain zero. As seen in Table 2, the goodput of normal priority traffic for each source is only about $0.2 \mathrm{Mb}$, instead of the traffic generation rate of $0.6 \mathrm{Mb}$. The reason is that

\section{QueueSizeVS.Time}

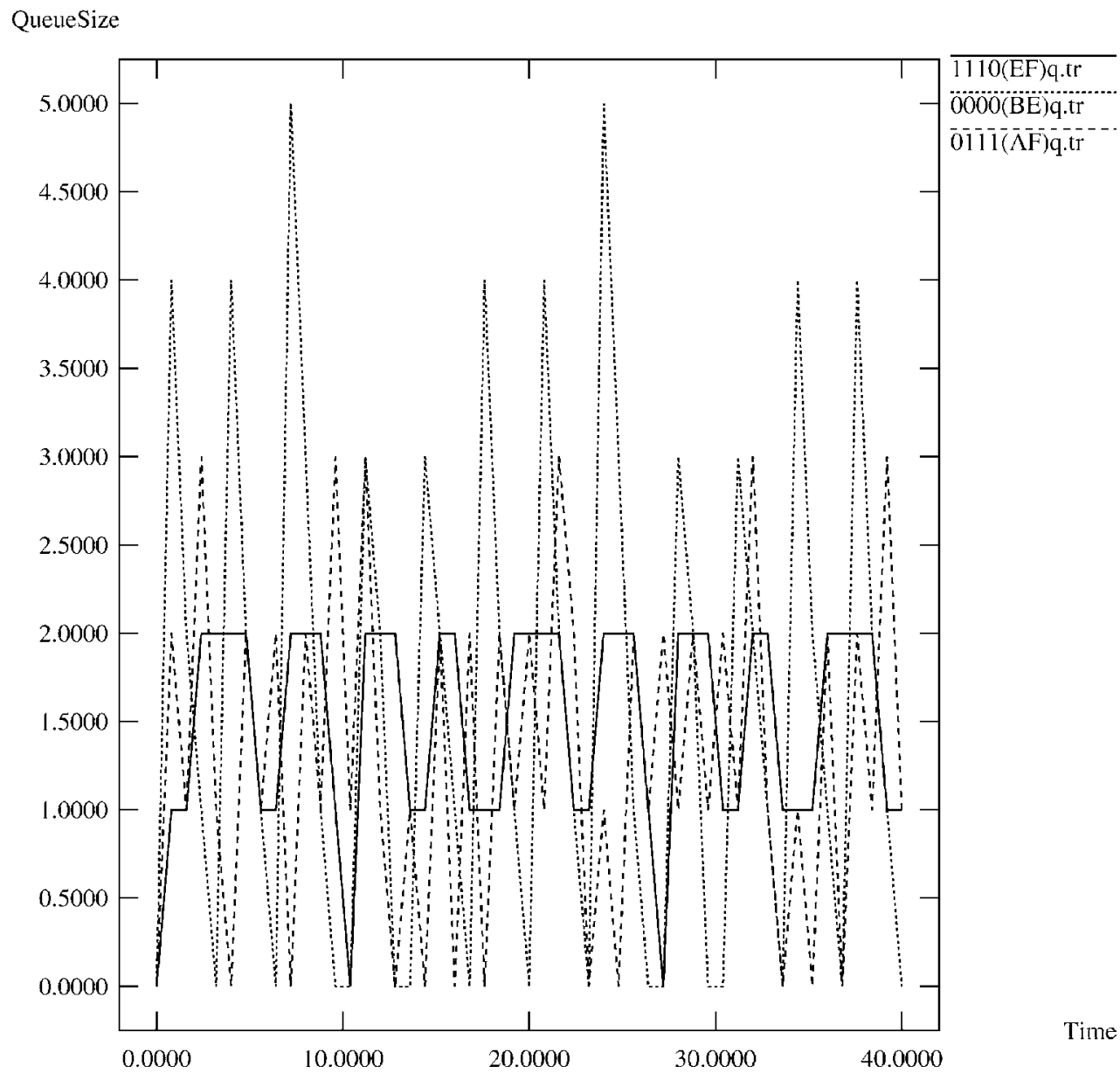

Fig. 7 Queue size for Case 1. 
the total available output bandwidth of normal priority traffic has been assigned to $1 \mathrm{Mb}$ by scheduler. From Fig. 8, we find that the average queue size of the normal priority queue is far greater than the other two types of sources. In addition, the jitter of normal priority traffic is also greater than the other two types of sources. The high priority traffic has the smallest average queue size and the smallest jitter.

Case 3 is very similar to Case 2 . The only difference is that the medium priority traffic, rather than normal priority traffic, gets into congestion. Though, in reality, it is less possible for medium priority traffic than normal priority traffic to get into congestion, we still use this case to test the performance of AF11 queue in CLNP-IP router and the effectiveness of our proposed scheme. As expected, we find the drop ratio of medium priority traffic is increased with the other two types of traffic remaining zero, and the goodput is also limited by the output link bandwidth assigned by the scheduler (which is $2 \mathrm{Mb}$ ). From Fig. 9, we find that both the jitter and the average queue size of medium priority traffic are far greater than the other two traffic types. Though this observation is rare in reality, it is still reasonable in this simulation case that the average queue size of the medium priority traffic is greater than the normal priority traffic. Because, for the purpose we have described, we artificially make the medium priority traffic congested, however, the normal priority traffic is not congested. The high priority traffic has the smallest average queue size and the smallest jitter.

\section{QueueSizeVS.Time}

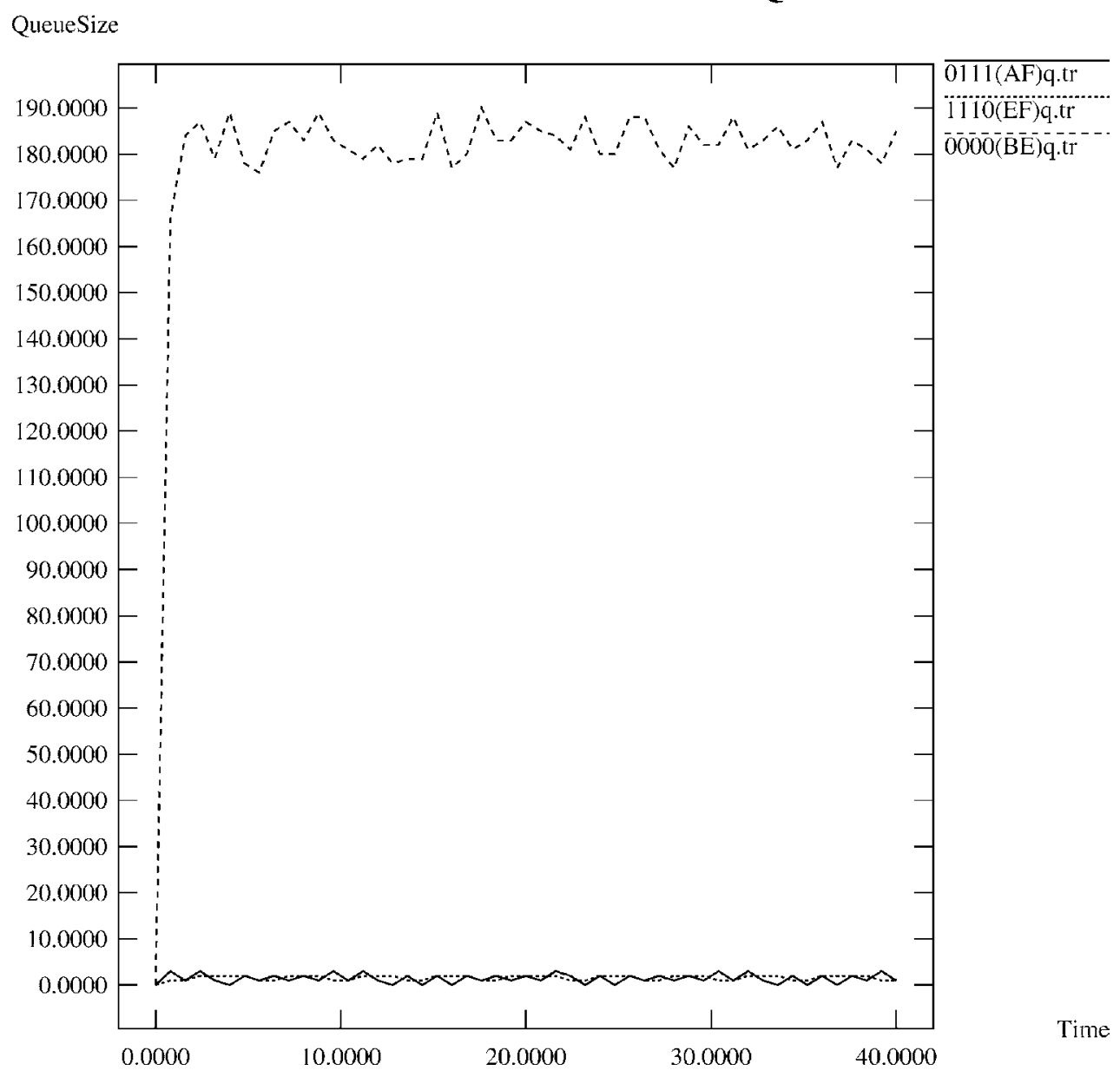

Fig. 8 Queue size for Case 2. 


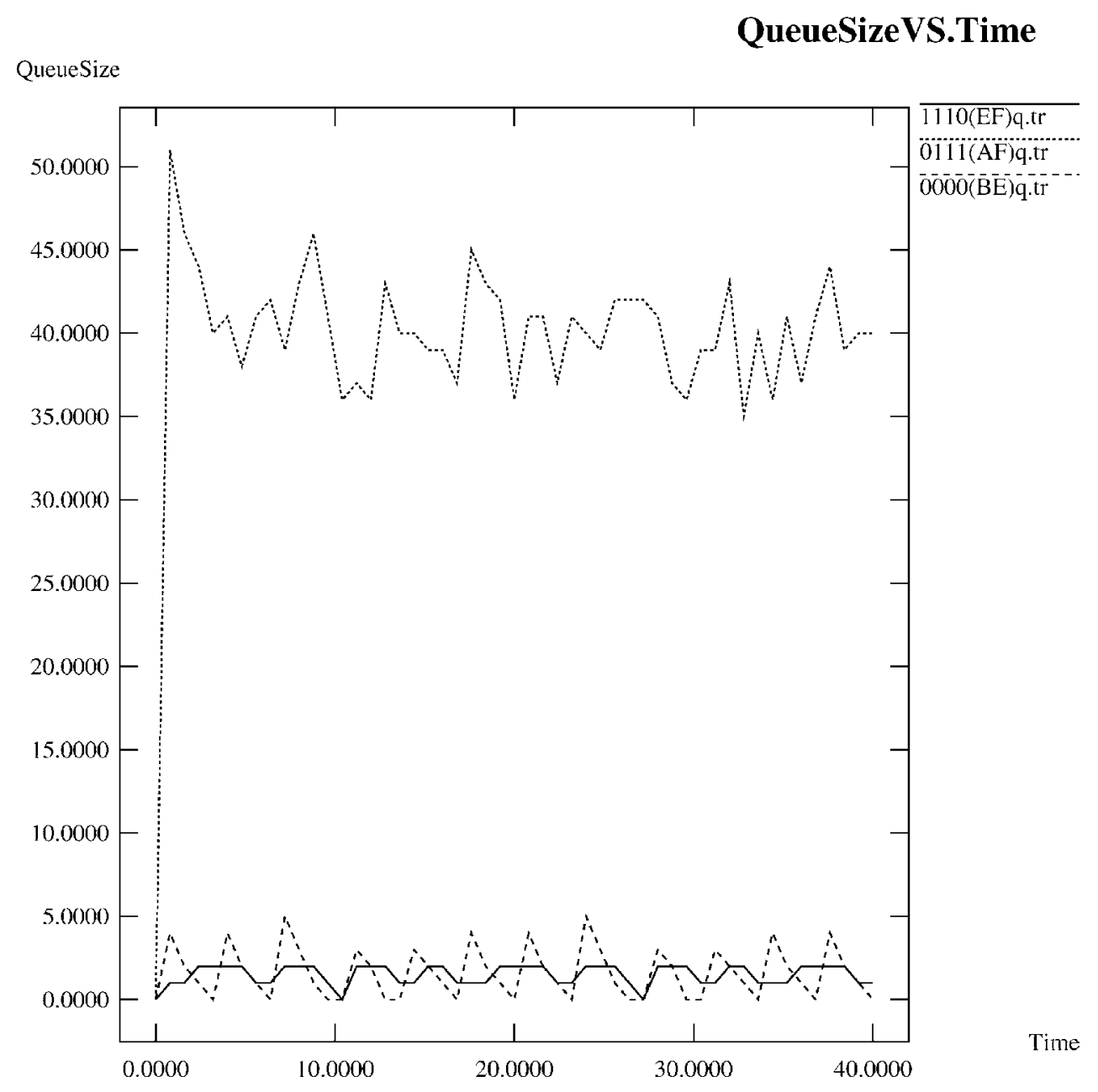

Fig. 9 Queue size for Case 3.

In Case 4, we increased the traffic generating rates of both medium and normal priority sources. Both of them get into network congestion in this case. We find from Table 3 that the drop ratio of high priority traffic remains zero, and drop ratios of both medium priority traffic and normal priority traffic are greatly increased. Furthermore, the drop ratio of normal priority traffic is greater than that of medium priority traffic. As shown by Table 2 , the goodput of both the medium and normal priority traffic are limited by their link bandwidths allocated by scheduler. From Fig. 10, we see that the normal priority traffic has both the biggest jitter and biggest average queue size. We can also find that the high priority traffic has both the smallest jitter and smallest average queue size.

From the results of above four cases, we summarize our observations as follows:

- The high priority traffic always has the smallest jitter, the smallest average queue size and the smallest drop ratio without being affected by the performance of other traffic. In other words, the high priority traffic receives the highest priority, which satisfies the priority requirements of ATN.

- The medium priority traffic has smaller drop ratio, jitter and queue size than the normal priority traffic, even in the presence of network congestion. This also satisfies the priority requirements of ATN.

We therefore, conclude that the priority requirements of ATN can be successfully achieved when ATN traffic is mapped to the DiffServ domain in next generation Internet. 


\section{QueueSizeVS.Time}

QueueSize

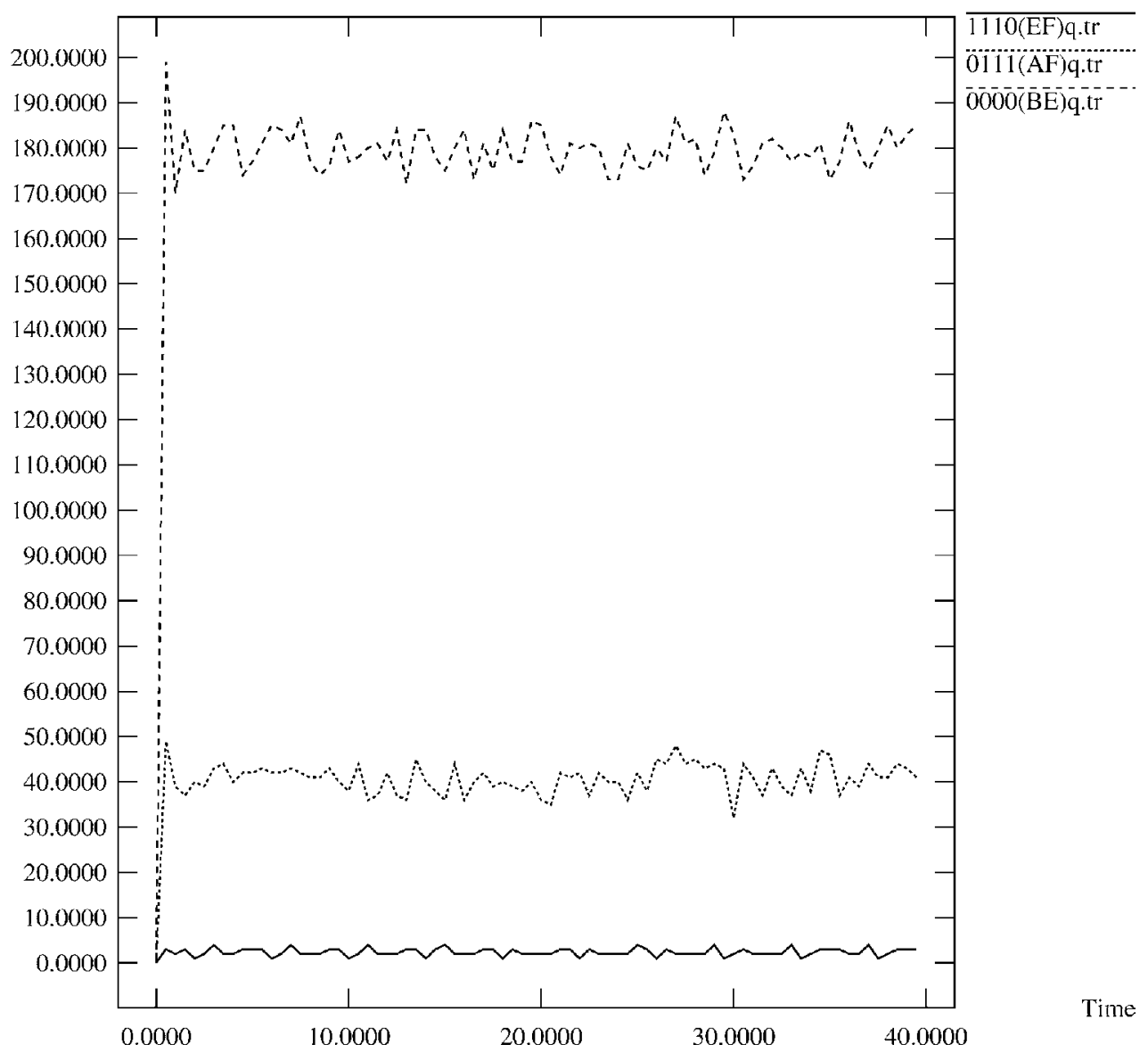

Fig. 10 Queue size for Case 4.

\section{Conclusions}

In this paper, we have proposed DiffServ as the network backbone for ground-ground communication to interconnect ATN air-ground networks. As one of the strategies, recommended by ARINC 664 Part 8, to inter-operate ATN and TCP/IP networks, a CLNP-IP router architecture with a mapping function have been developed to map traffic flows coming from ATN with different priorities (indicated by the priority field in ATN packet header) to the corresponding services in the DiffServ domain.

The proposed scheme has been evaluated in detail using simulations. It has been found that the QoS requirements of ATN can be satisfied by the proposed architecture. We validated this by measuring the drop ratio, goodput and queue size. Simulation results showed that high priority ATN traffic had the smallest jitter, the smallest average queue size, and the smallest drop ratio, and was unaffected by the performance of other traffic. Moreover, medium priority ATN traffic had a smaller drop ratio, jitter and queue size than the normal traffic, even in the presence of network congestion.

\section{References}

${ }^{1}$ ATNP, “ATN SARPs—2 $2^{\text {nd }}$ Edition,” Final Editor's drafts of the ATN SARPs, December 1999.

${ }^{2}$ Braden, R., Clark, D., and Shenker, S., “Integrated Services in the Internet Architecture: an Overview,” RFC 1633, June 1994. 
${ }^{3}$ Blake, S., Black, D., Carlson, M., Davies, E., Wang, Z., and Weiss, W., “An Architecture for Differentiated Services,” RFC 2475, December 1998.

${ }^{4}$ AEEC ADN committee, "ARINC 664 Aircraft Data Network, Part 8-Interoperation with non-IP Protocols and Services," Draft 3, January 2005.

${ }^{5}$ Smith, P., "IPSKY: IPv6 for the aeronautical telecommunications network," $20^{\text {th }}$ Digital Avionics Systems Conference, Daytona Beach, FL, October 2001, pp. 7A6/1-7A6/11.

${ }^{6}$ Dhas, C. and Wargo, C., "Potential use of Internet protocols in Aviation," IEEE Aerospace Conference, Big Sky, MT, March 2001, pp. 3/1345-3/1357.

${ }^{7}$ Netto, C. and Khanna, M., “ATN over IP Models for Evaluation,” The $22^{\text {nd }}$ Digital Avionics Systems Conference, October 2003, pp. 4.B.4-41-10.

${ }^{8}$ Crpuzard, L., Gawinowski, G., Robert, O., and Smith, P., "IPv6 and Internet Technology for the Aeronautical Telecommunication Network," The $3^{\text {rd }}$ USA/Europe Air Traffic Management Seminar, Napoli, Italy, June 2000.

${ }^{9}$ ATNP, “Comprehensive ATN Manual (CAMAL)," Final Editor's drafts of the ATN Guidance Material, January 1999.

${ }^{10}$ Hof, H., "Change Proposal for Improved Text on the ATN Priority Architecture," ATNP/WG2/WP174, October 1995.

${ }^{11}$ Nichols, K., Blake, S., Baker, F., and Black, D., "Definition of the Differentiated Services Field (DS Field) in the IPv4 and IPv6 Headers," RFC 2474, December 1998.

${ }^{12}$ Bernet, Y., Blake, S., Grossman, D., and Smith, A., "An Informal Management Model for Diffserv Routers," draft-ietfdiffserv-model-04.txt, July 2000.

${ }^{13}$ Jacobson, V., Nichols, K., and Poduri, K., “An Expedited Forwarding PHB,” RFC 2598, June 1999.

${ }^{14}$ Heinanen, J., Baker, F., Weiss, W., and Wroclawski, J., “Assured Forwarding PHB Group,” RFC 2597, June 1999.

${ }^{15}$ Floyd, S. and Jacobson, V., "Random Early Detection gateways for Congestion Avoidance," IEEE/ACM Transactions on Networking, Vol. 1, No. 4, August 1993, pp. 397-413.

Reinaldo Perez

Associate Editor 